The Chittagong Univ. J. Sci. 40 : 75-96, 2018

\title{
Effect of Fukushima Accident on Fishes and Fish Like other Foods Collected from the Bay of Bengal and Karnaphuli River, Bangladesh
}

\author{
Akhi Das Gupta ${ }^{1}$, Md. Kowsar Alam ${ }^{1}$, Shyamal Ranjan Chakraborty ${ }^{1 *}$, \\ A. K. M. Rezaur Rahman ${ }^{1}$, S. I. Bhuian ${ }^{2}$, Masud Kamal ${ }^{2}$, \\ Hasan Murad ${ }^{1}$ and Arun Kumar Deb ${ }^{1}$ \\ ${ }^{1}$ Department of Physics, University of Chittagong, Chittagong-4331, Bangladesh \\ ${ }^{2}$ Bangladesh Atomic Energy Commission, Chittagong, Bangladesh \\ *Corresponding author: shyamal@cu.ac.bd
}

\begin{abstract}
An experiment was carried out to assess the radiological exposure due to the intake of fishes and fish like foods from some common estuary (Karnaphuli River) and Marine (Bay of Bengal) contaminated by Fukushima Reactor Accident, if any, by using gamma spectroscopy. The analysis was done to estimate the radioactivity concentrations due to natural radionuclides namely ${ }^{238} \mathrm{U},{ }^{232} \mathrm{Th}$ and ${ }^{40} \mathrm{~K}$ and artificial radionuclide ${ }^{137} \mathrm{Cs}$ in Karnaphuli estuary and Chittagong city adjoining Bay of Bengal fish and fish-like samples. The activity concentration for ${ }^{238} \mathrm{U}$ in all the samples ranged from $0.10615 \pm 0.0000$ to $2.4767 \pm 0.0005 \mathrm{~Bq} \cdot \mathrm{kg}^{-1}$ with the mean of $0.6109 \pm 0.0001 \mathrm{~Bq} \cdot \mathrm{kg}^{-1}$. The Activity of ${ }^{232} \mathrm{Th}$ ranged from $0.0009 \pm$ 0.0000 to $0.0273 \pm 0.0000 \mathrm{~Bq} \cdot \mathrm{kg}^{-1}$ with the mean of $0.0074 \pm 0.0000 \mathrm{~Bq} \cdot \mathrm{kg}^{-1}$. The activity concentration for ${ }^{40} \mathrm{~K}$ was found to be in the range from $1.5516 \pm 0.0311$ to $74.8658 \pm 0.2532 \mathrm{~Bq} \cdot \mathrm{kg}^{-1}$ with the mean of $24.9533 \pm 0.0702 \mathrm{~Bq} \cdot \mathrm{kg}^{-1}$. The artificial radionuclide ${ }^{137} \mathrm{Cs}$ was not found in any of the samples. The specific activity values for most of the sample were within the permissible limits. The average effective dose and internal hazard index due to consumption of those fishes and fish-like
\end{abstract}


76 Akhi Das Gupta, Md. Kowsar Alam, Shyamal Ranjan Chakraborty, A. K. M. Rezaur Rahman, S. I. Bhuian, Masud Kamal, Hasan Murad and Arun Kumar Deb

foods was found to be $0.1 \mathrm{mSv} \cdot \mathrm{y}^{-1}$ and $0.0085 \pm 0.0000 \mathrm{mSv} \cdot \mathrm{y}^{-1}$ respectively. The research work showed that the consumers of concerned fishes and fish-like foods of the Chittagong area have no risk of radioactivity ingestion even though no amount of radiation is assumed to be totally safe.

Keywords: River Fishes; Sea Fishes; Ingestion; Radioactive Exposure.

এ গবেষণা কর্মটি ফুকুশিমা পরমাণু চুল্লী দূর্ঘটনা জনিত কারণে দূষিত, যদি হয়ে থাকে, নদী (কর্ণফুলী) ও সমুদ্রের (বঙ্গোপসাগর) সাধারণ মোহনার মাছ ও মাছের মতো খাদ্য গ্রহণের নিমিত্তে তেজস্ক্রিয় সম্পাত নির্ণয়ের উস্দেশ্যে গামা বর্ণালীমিতির সাহায্যে সম্পাদন করা হয়েছিল। কর্ণফুলী নদীর মোহনা ও চট্টগ্রাম নগর সংলগ্ন বঙ্গোপসাগরের মাছ ও মাছের মতো নমুনায় প্রাকৃতিক তেজক্ক্রিয় নিউক্লিয়াস সমূহ ${ }^{238} \mathrm{U},{ }^{232} \mathrm{Th}$ ও ${ }^{40} \mathrm{~K}$ এবং কৃত্রিম তেজস্ক্রিয় নিউক্লিয়াস ${ }^{137} \mathrm{Cs}$-এর ঘনত্ব নির্ণয়ে বিশ্লেষণ করা হয়েছিল। নমুনা সমূহে ${ }^{238} \mathrm{U}$-এর তেজস্ক্রিয় ঘনত্বের গড় ০.৬১০৯ \pm ০.০০০১ বেকরেল.কেজি-১ ০.১০৬১৫ \pm 0.0000 হতে ২.৪৭৬৭ $\pm 0.000 ৫$ বেকরেল.কেজিপরিসরের মধ্যে পাওয়া গিক়েছিল। তেজস্ক্রিয় নিউক্লিয়াস ${ }^{232} \mathrm{Th}$-এর সক্রিয়তা ০.০০০৯ \pm

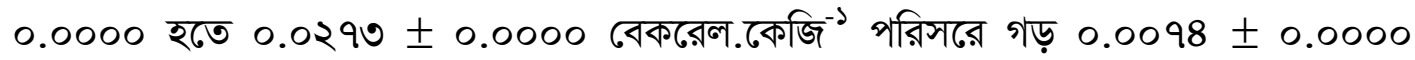
বেকরেল.কেজি-১ পাওয়া গিক্যেছি। তেজস্ক্রিয় নিউক্লিয়াস ${ }^{40} \mathrm{~K}$-এর সক্রিয়তা ঘনত্ব ১.৫৫১৬ \pm

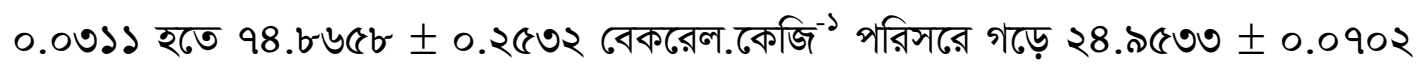
বেকরেল.কেজি-১ পাওয়া গিয়েছিল । কৃত্রিম তেজস্ক্রিয় নিউক্লিয়াস ${ }^{137} \mathrm{Cs}$-এর অস্তিত্ব কোন নমুনায় পাওয়া যায়নি। আপেক্ষিক সক্রিয়তার মান প্রায় সবগুলো নমুনায় অনুমোদিত মাত্রার মধ্যে গিয়েছিল। এ মাছ ও মাছের মতো খাদ্য গ্রহনের ফলে গড় সক্রিয় দাগ ও আভ্যন্তরীণ বিপদ সূচক 
Effect of Fukushima Accident on Fishes and Fish Like other Foods Collected from the Bay of Bengal and Karnaphuli River, Bangladesh

যথাক্রমে ০.১ মিলি-সিভার্ট.বৎসর ${ }^{-১}$ ও ০.০০b৫ \pm ০.০০০০ পাওয়া গিয়েছিল। এ গবেষণা থেকে দৃষ্ট হয় যে চট্টগাম অঞ্চলের ভোক্তারা সংশ্লিষ্ট মাছ ও মাছের-মতো খাদ্য গ্রহণের ফলে তেজস্ক্রিয়তা গলাধঃকরণের ঝুঁকিতে নেই যদিও কোন মাত্রার বিকিরণই সম্পূর্ণরূপে নিরাপদ নয় বলে মনে করা হয়।

\section{Introduction}

Radioactivity up to a certain level has always been present in the environment since the formation of the earth [1]. The level of radioactivity in the environment has begun to increase continually with the use of radioisotopes in medicine, industry, testing of nuclear weapons, nuclear accidents and nuclear power plants for nuclear energy. Radiation damage is severe in the case of acute exposure, than in the case of chronic exposure. This means that the human body is able to recuperate and repair radiation damage which is kept within certain limits and below certain threshold. There is no way to prevent the natural radiation exposure completely and absolutely. The purpose of the radiation protection is to reduce the radiation doses received by general masses to below the threshold amount which would induce permanent injury [2]. The radioactive substances which enter into the body are among the most insidious threat to living tissue. Radionuclides present in the atmosphere reach the ground level, also in the sea and river water principally through rain and dry deposition. Finally they enter into the human body mainly through food and water, i.e. ingestion and inhalation [3]. 
78 Akhi Das Gupta, Md. Kowsar Alam, Shyamal Ranjan Chakraborty, A. K. M. Rezaur Rahman, S. I. Bhuian, Masud Kamal, Hasan Murad and Arun Kumar Deb

Bangladesh is a country of enormous natural beauty having nationwide river, large coastal area, water lakes, etc. The climate condition in Bangladesh makes it possible to grow huge variety of fishes. Sources of fishes here are the sea, rivers, canals, lakes, open wetlands and ponds. Fish is a food of excellent nutritional value, providing high quality and easily digestible protein and a wide variety of vitamins and minerals. Fishes have a significant positive impact on improving the quality of dietary protein by complementing the essential amino acids that are often present in low quantities in vegetable-based diets. Fish oils in fatty fish are the richest source of a type of fat that is vital to normal brain development in unborn babies and infants. Without adequate amounts of these fatty acids, normal brain development does not take place [4-6]. Bangladeshi people usually like to keep fish in their daily menu of foods.

According to nutrition scientist an adult people should consume about $0.8 \mathrm{~g} \cdot \mathrm{kg}^{-1}$ (body mass) proteins daily [7, 8]. Among them $15.1 \mathrm{~g}$ will be animal protein. For Bangladeshi people $80 \%$ of animal protein comes from fishes [9] Fish is popular accepted protein because it can widely preserve through drying, salting, freezing and canning. This makes fish a food of choice not only to the Bangladeshi people but many other people of the world. About $12 \%$ of Bangladeshi people are directly or indirectly engaged with fish and fish related jobs and business [9]. People earn their livelihood by catching fish from open stream, rivers or deep sea. The position of fish is in third for earning foreign currency to Bangladesh. About $10 \%$ of total export income comes from the fish and fish products [10]. 
Due to serious nuclear accident in Fukushima, Japan in March 2011 a large amount of radionuclides $\left({ }^{131} \mathrm{I},{ }^{134} \mathrm{Cs},{ }^{137} \mathrm{Cs}\right)$ went into the Pacific Ocean [11 - 13]. The present study is to investigate the effect of this huge radiation in the sea-foods of Bay of Bengal.

\section{Materials and Methods}

\subsection{Counting Efficiencies of the HPGe Detector}

The counting efficiency of the detector was calculated using the following formula $[14,15]$.

$$
\text { Efficiency }=\frac{C P S}{\text { Activity } \times \text { Intensity }}
$$

where,

CPS $=$ Net count per second (i.e. CPS from standard source - CPS for background)

Activity $(A)=$ Standard source activity for respective energy

Intensity $\left(I_{\gamma}\right)=$ Intensity of gamma energies.

\subsection{Data acquisition and analysis for Activity calculation}

The samples after pretreatment, preparation and packing in the air tight sealed pots have been stored for 4 weeks to reach secular equilibrium between the ${ }^{238} \mathrm{U}$ and ${ }^{232} \mathrm{Th}$ series and their respective progeny before their measurements $[15,16]$. It is assumed that ${ }^{222} \mathrm{Rn}$ and ${ }^{220} \mathrm{Rn}$ could not escape from the sealed containers after closure. The gamma ray activities of the fish samples have been in High Purity Germanium Detector (HPGe) that has been coupled with Digital Spectrum Analyzer-1000 (DSA-1000). For calculating the counting efficiencies and hence the 
80 Akhi Das Gupta, Md. Kowsar Alam, Shyamal Ranjan Chakraborty, A. K. M. Rezaur Rahman, S. I.

Bhuian, Masud Kamal, Hasan Murad and Arun Kumar Deb

activity of samples, the adjustment of necessary parameters of the detector such as resolution, peak to Compton ratio etc and measurement of minimum detectable activity of the detector have been done. Each of the standard source and collected samples have been placed on the top of the detector one by one in due courses within the shielding arrangement by following the standard procedure. The standard sources samples have been counted for 55,160 seconds and each of the collected samples have been counted for 10,000 seconds. The most prominent gamma ray energy peaks have been found to be $[3,15,17]$ at $238.63 \mathrm{keV}$ (due to ${ }^{212} \mathrm{~Pb}$ ); $727.17 \mathrm{keV}$ (due to ${ }^{212} \mathrm{Bi}$ ); $295.21 \mathrm{keV} \& 351.92 \mathrm{keV}$ (due to ${ }^{214} \mathrm{~Pb}$ ); 338.40 $\mathrm{keV}, 911.07 \mathrm{keV} \& 969.11 \mathrm{keV}$ (due to ${ }^{228} \mathrm{Ac}$ ); $510.57 \mathrm{keV}, 583.19 \mathrm{keV} \& 2614.53$ $\mathrm{keV}$ (due to ${ }^{208} \mathrm{Tl}$ ); $609.31 \mathrm{keV} \& 1764.49 \mathrm{keV}$ (due to ${ }^{214} \mathrm{Bi}$ ); $1460.75 \mathrm{keV}$ (due to ${ }^{40} \mathrm{~K}$ ) and $661.66 \mathrm{keV}$ (due to ${ }^{137} \mathrm{Cs}$ ). Activities of the natural radionuclides presented in the fish samples have been calculated by using the following formula [18]:

$$
A=\frac{C P S \times 1000}{\varepsilon(a b s) \times I_{\gamma} \times W}
$$

Where, $\mathrm{A}$ is the activity in Bq. $\mathrm{kg}^{-1}$,

CPS is the net count per second of the experimental sample,

$\mathrm{W}$ is the weight of the sample in gm, $\varepsilon(\mathrm{abs})$ is the absolute counting efficiency of the detector, and $\mathrm{I}_{\gamma}$ is the absolute gamma intensity of the corresponding gamma ray energy. The error of the measurements was expressed in terms of standard deviation $( \pm \sigma)$, when, $\sigma=\sqrt{\bar{x}}$; where, $x=$ Activity of radionuclides. 


\subsection{Preparation of Samples}

In this study, a total number of 14 samples have been collected from four locations of Chittagong City namely (1) FishariGhat (shipping fishing zone), (2) Reazuddin Bazar, (3) Chawk Bazar and (4) Directly from fisherman of "fifteen no. Naval Ghat" by ensuring that the fishing have been done on the Karnaphuli estuary or/and the Bay of Bengal. The samples were collected in a clean polyethylene bag and proper identification number was given. All the samples were divided into two parts like crab and flesh after that the samples were prepared for taking the counting $[19,20]$.

\subsection{Samples collected}

Samples collected from the Karnaphuli Samples collected from the sea (the Bay Estuary of Bengal)

1. Crab (Epibolocera sinuatifrons), 1. Surma (Rastrelliger kanagurta),

2. Chingri (Macrobrachium carcinus), 2. Baila (Awaous guamensis),

3. Shing (Gangata youssoufi),

3. Chanda (Pampus argenteus),

4. Hilsha (Tenualosa ilisha),

4. Powa (Otolihes argentues),

5. Tengra (Nemapteryx nenga),

5. Loytta (Harpodon nehereus),

6. Gura Chingri (Palaemon styliferus), and

6. Mola (Amblypharyngodon mola), and

7. Tailla (Eleutheronema tetradactylum).

7. Chandan Ilish (Hilsa kanagurta).

\subsection{Calculation of Radiological parameters}

\subsubsection{Dose estimations from annual intakes}

The risk associated with an intake of radionuclides in the body is proportional to 
82 Akhi Das Gupta, Md. Kowsar Alam, Shyamal Ranjan Chakraborty, A. K. M. Rezaur Rahman, S. I.

Bhuian, Masud Kamal, Hasan Murad and Arun Kumar Deb

the total dose delivered by the radionuclides while staying in the various organs. In general it is assumed that stochastic effects occur linearly with dose and usually the effective dose equivalent is used to define the risk. So, effective dose equivalent is a parameter for the biological effect. Intakes to effective dose equivalent conversion factors are needed in order to convert the intake into dose on ingestion of radionuclides into the body. The intake to dose conversion factors (50 years period) cited in the ICRP publication no. 51 for the members of the public (adults) was used [21]. Radioactivity levels in fish were used to estimate internal effective doses. The effective dose to an individual from an intake of a radionuclide via ingestion of fish is calculated by this formula [21, 22],

$$
D_{\text {ing }}=C_{R} \times I_{F} \times E_{D}
$$

where,

$D_{\text {ing }}$ is the annual effective dose to an individual due to ingestion of radionuclides $\left(\mathrm{Sv} \cdot \mathrm{y}^{-1}\right)$

$C_{R}$ is the concentration of radionuclides in ingested fish (Bq. $\mathrm{kg}^{-1}$ )

$I_{F}$ is the annual intake of fish containing radionuclide's $\left(\mathrm{kg} . \mathrm{y}^{-1}\right)$ and the intake rates for Bangladeshi were taken from fish consumption statistics data [22, 23].

And, $E_{D}$ is the ingestion dose conversion factor for radionuclides ( $\left.\mathrm{Sv} \cdot \mathrm{Bq}^{-1}\right)$

Therefore, the total dose via ingestion is calculated by this formula,

$$
\begin{gathered}
D_{\text {ing }}=\left[C_{R}\left({ }_{92}^{238} U\right) \times I_{F} \times E_{D}\right]+\left[C_{R}\left({ }_{90}^{232} T h\right) \times I_{F} \times E_{D}\right] \\
+\left[C_{R}\left({ }_{19}^{40} K\right) \times I_{F} \times E_{D}\right]
\end{gathered}
$$




\subsubsection{Internal hazard index, $\mathrm{H}_{\mathrm{int}}$}

The internal hazard index $\left(\mathrm{H}_{\mathrm{int}}\right)$ gives the internal exposure to carcinogenic radon in the fish samples and is given by equation $[22,24]$.

$$
\mathrm{H}_{\text {int }}=\frac{\mathrm{C}_{\mathrm{U}}}{185}+\frac{\mathrm{C}_{\mathrm{Th}}}{259}+\frac{\mathrm{C}_{\mathrm{K}}}{4810}
$$

The value of this index should be less than $1 \mathrm{mSv}^{-1} \mathrm{y}^{-1}$ in order for the radiation hazard to have negligible hazardous effects to the respiratory organs of the public $[22,24]$.

\section{Results and Discussion}

\subsection{Activity of ${ }^{238} \mathrm{U}$}

The activity concentration of ${ }^{238} U$ has been found in the range from $0.10615 \pm 0$ to $2.4767 \pm 0.0005 \mathrm{~Bq} \cdot \mathrm{kg}^{-1}$ with the mean of $0.6109 \pm 0.0001 \mathrm{~Bq} \cdot \mathrm{kg}^{-1}$. The comparative data of the activity concentration of ${ }^{238} U$ of all samples is shown in table 1 and their corresponding graphical comparison is shown in figure 1 . The comparison of the results of the mean activity concentration of ${ }^{238} \mathrm{U}$ with the worldwide value and other study of the world is shown in table 2 and graphically in figure 2. The graphical comparison of the mean activity concentration of ${ }^{238} \mathrm{U}$ for river and sea samples is given in figure 3 .

\subsection{Activity of ${ }^{232} \mathrm{Th}$}

In the fish samples, activity concentration of ${ }^{232} \mathrm{Th}$ was found to be in the range of $0.000858 \pm 0$ and $0.0273 \pm 0 \mathrm{~Bq} \cdot \mathrm{kg}^{-1}$, with a mean value $0.0074 \pm 0 \mathrm{~Bq} \cdot \mathrm{kg}^{-1}$. The activity concentration of ${ }^{232} \mathrm{Th}$ of all samples is shown in table 1 with a 
84 Akhi Das Gupta, Md. Kowsar Alam, Shyamal Ranjan Chakraborty, A. K. M. Rezaur Rahman, S. I. Bhuian, Masud Kamal, Hasan Murad and Arun Kumar Deb

comparative graphical scheme in figure 4. The variation of the mean activity concentration of ${ }^{232} \mathrm{Th}$ of present study with the worldwide value and other study of the world is shown in table 2. The graphical comparison of the mean activity of ${ }^{232}$ Th of present study with the worldwide value and other study is shown in figure 2. The comparative pictorial representation of the mean activity concentration of ${ }^{232} \mathrm{Th}$ for river and sea samples is given in figure 5 .

\subsection{Activity of ${ }^{40} \mathrm{~K}$}

The mean specific activity of ${ }^{40} \mathrm{~K}$ in the fish samples has been found to be 24.9533 $\pm 0.0702 \mathrm{~Bq} \cdot \mathrm{kg}^{-1}$ having the range from $1.5516 \pm 0.0311$ to $74.8658 \pm 0.2532$ Bq. $\mathrm{kg}^{-1}$. The distribution of the activity concentrations of ${ }^{40} \mathrm{~K}$ of all samples is shown in table 1 and their corresponding graphical comparison is shown in figure 6. The comparison of the mean activity concentration of ${ }^{40} \mathrm{~K}$ with worldwide value and other study of the world has been shown in table 2 . The variation of the mean activity of ${ }^{40} \mathrm{~K}$ of the present study with the worldwide value and other study has been shown in figure 2. The comparative pictographic illustration of the mean activity concentration of ${ }^{40} \mathrm{~K}$ for river and sea samples is given in figure 7 .

\subsection{Activity of ${ }^{137} \mathrm{Cs}$}

The anthropogenic radionuclide ${ }^{137} \mathrm{Cs}$ was not found in any samples.

Table 1: The comparative data of the activity concentration of parent radionuclide's, ${ }^{40} \mathrm{~K}$ and ${ }^{137} \mathrm{Cs}$ of all fish samples 
Effect of Fukushima Accident on Fishes and Fish Like other Foods Collected from the Bay of Bengal and Karnaphuli River, Bangladesh

\begin{tabular}{|c|c|c|c|c|c|}
\hline $\begin{array}{l}\text { Sample } \\
\text { ID }\end{array}$ & $\begin{array}{c}\text { Activity } \\
\text { Concentration } \\
\text { of }{ }^{238} \mathrm{U} \\
\left(\mathrm{Bq} \cdot \mathrm{kg}^{-1}\right) \text { with } \\
( \pm 1 \sigma)\end{array}$ & $\begin{array}{c}\text { Activity } \\
\text { Concentration } \\
\text { of }^{232} \mathrm{Th} \\
\left(\mathrm{Bq} \cdot \mathrm{kg}^{-1}\right) \text { with } \\
( \pm 1 \sigma)\end{array}$ & $\begin{array}{c}\text { Activity } \\
\text { Concentration } \\
\text { of }{ }^{40} \mathrm{~K} \\
\left(\mathrm{~Bq} \cdot \mathrm{kg}^{-1}\right) \text { with } \\
( \pm 1 \sigma)\end{array}$ & $\begin{array}{c}\text { Activity } \\
\text { Concentration } \\
\text { of }{ }^{137} \mathrm{Cs} \\
\left(\mathrm{Bq} \cdot \mathrm{kg}^{-1}\right) \text { with } \\
( \pm 1 \sigma)\end{array}$ & $\begin{array}{c}\text { Total activity } \\
\text { concentration } \\
\text { in }\left(\mathrm{Bq}_{\mathrm{kg}}^{-1}\right) \\
\text { with }( \pm 1 \sigma)\end{array}$ \\
\hline $\mathrm{R}-01$ & $\begin{array}{c}0.2328 \pm \\
0.0001\end{array}$ & $0.0034 \pm 0$ & $\begin{array}{c}1.7646 \pm \\
0.0437\end{array}$ & ND & $2.0001 \pm 0.04$ \\
\hline R-02 & $\begin{array}{c}2.4767 \pm \\
0.0005\end{array}$ & $0.0273 \pm 0$ & $\begin{array}{c}74.8658 \pm \\
0.2532\end{array}$ & ND & $77.3698 \pm 0.25$ \\
\hline R-03 & $\begin{array}{c}0.7170 \pm \\
0.0002\end{array}$ & $0.0086 \pm 0$ & $\begin{array}{c}55.0649 \pm \\
0.0796\end{array}$ & ND & $55.7905 \pm 0.08$ \\
\hline R-04 & $\begin{array}{c}0.1706 \pm \\
0.0000\end{array}$ & $0.0019 \pm 0$ & $\begin{array}{c}15.3436 \pm \\
0.0233\end{array}$ & ND & $15.5161 \pm 0.02$ \\
\hline R-05 & $\begin{array}{l}1.5038 \pm \\
0.0003\end{array}$ & $0.0203 \pm 0$ & $\begin{array}{c}29.3928 \pm \\
0.1778\end{array}$ & ND & $30.9169 \pm 0.18$ \\
\hline R-06 & $\begin{array}{c}0.7274 \pm \\
0.0002\end{array}$ & $0.0101 \pm 0$ & $\begin{array}{c}48.2191 \pm \\
0.0882\end{array}$ & ND & $48.9566 \pm 0.09$ \\
\hline $\mathrm{R}-07$ & $\begin{array}{c}0.8303 \pm \\
0.0002\end{array}$ & $0.0075 \pm 0$ & $\begin{array}{c}47.3487 \pm \\
0.1028\end{array}$ & ND & $48.1865 \pm 0.10$ \\
\hline S-01 & $0.1062 \pm 0$ & $0.0010 \pm 0$ & $\begin{array}{c}11.6636 \pm \\
0.0156\end{array}$ & ND & $11.7708 \pm 0.02$ \\
\hline S-02 & $\begin{array}{c}0.1917 \pm \\
0.0001\end{array}$ & $0.0039 \pm 0$ & $\begin{array}{c}1.5516 \pm \\
0.0311\end{array}$ & ND & $1.7472 \pm 0.03$ \\
\hline S-03 & $0.1653 \pm 0$ & $0.0009 \pm 0$ & $2.1360 \pm 0$ & ND & $2.3022 \pm 0$ \\
\hline S-04 & $\begin{array}{c}0.3022 \pm \\
0.0001\end{array}$ & $0.0033 \pm 0$ & $\begin{array}{c}20.3025 \pm \\
0.0287\end{array}$ & ND & $20.6080 \pm 0.03$ \\
\hline S-05 & $\begin{array}{c}0.5007 \pm \\
0.0001\end{array}$ & $0.0072 \pm 0$ & $\begin{array}{c}15.5568 \pm \\
0.0636\end{array}$ & ND & $16.0647 \pm 0.06$ \\
\hline S-06 & $\begin{array}{c}0.4495 \pm \\
0.0001\end{array}$ & $0.0058 \pm 0$ & $\begin{array}{c}9.2995 \pm \\
0.0493\end{array}$ & ND & $9.7548 \pm 0.05$ \\
\hline S-07 & $\begin{array}{c}0.1784 \pm \\
0.0001\end{array}$ & $0.0025 \pm 0$ & $\begin{array}{c}16.8369 \pm \\
0.0263\end{array}$ & ND & $17.0178 \pm 0.03$ \\
\hline Mean & $\begin{array}{c}0.6109 \pm \\
0.0001\end{array}$ & $0.0074 \pm 0$ & $\begin{array}{c}24.9533 \pm \\
0.0702\end{array}$ & ND & $25.5716 \pm 0.07$ \\
\hline
\end{tabular}

$\mathrm{R} \rightarrow$ River, $\mathrm{S} \rightarrow$ Sea 
86 Akhi Das Gupta, Md. Kowsar Alam, Shyamal Ranjan Chakraborty, A. K. M. Rezaur Rahman, S. I. Bhuian, Masud Kamal, Hasan Murad and Arun Kumar Deb

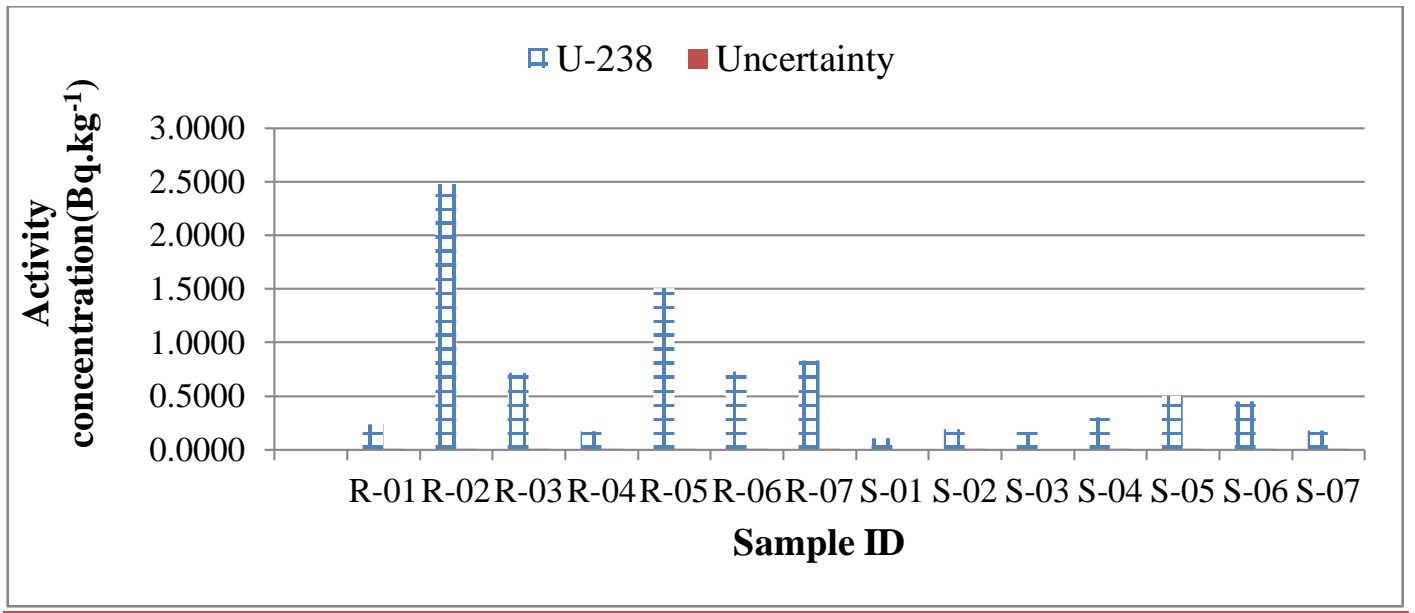

Fig. 1: Comparison of the activity concentrations of ${ }^{238} U$ of all the fish samples.

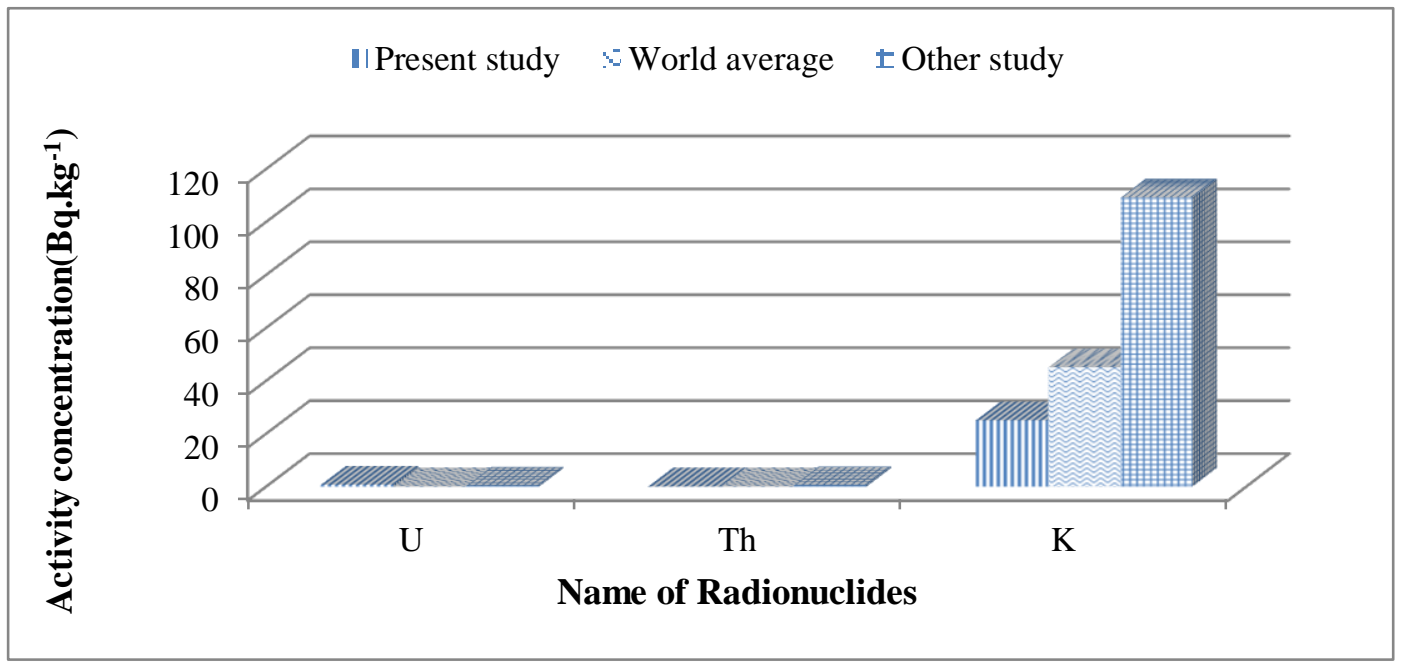

Fig. 2: Comparison of the mean activity concentration (Bq. $\mathrm{kg}^{-1}$ ) of parent radionuclides, and ${ }^{40} \mathrm{~K}$ of all fish samples with world average and other study. 
Effect of Fukushima Accident on Fishes and Fish Like other Foods Collected from the Bay of Bengal and Karnaphuli River, Bangladesh

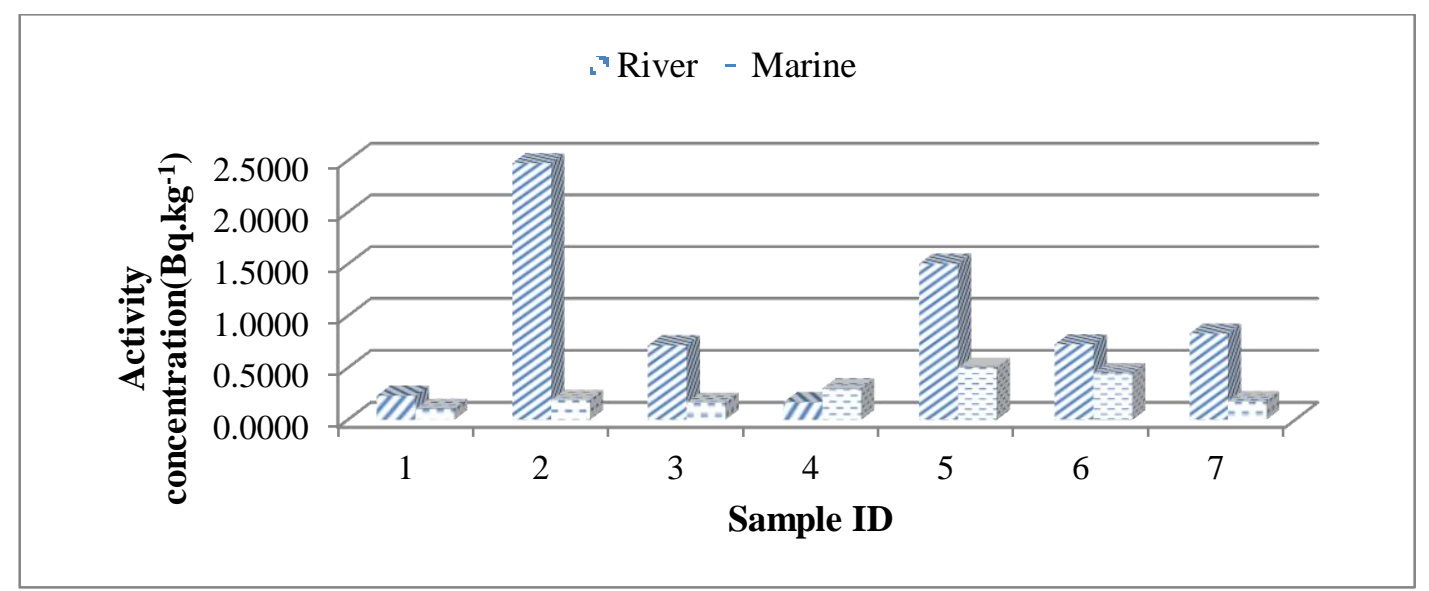

Fig. 3: Comparison of activity concentration of ${ }^{238} \mathrm{U}$ between river (Karnaphuli estuary) and marine fish samples.

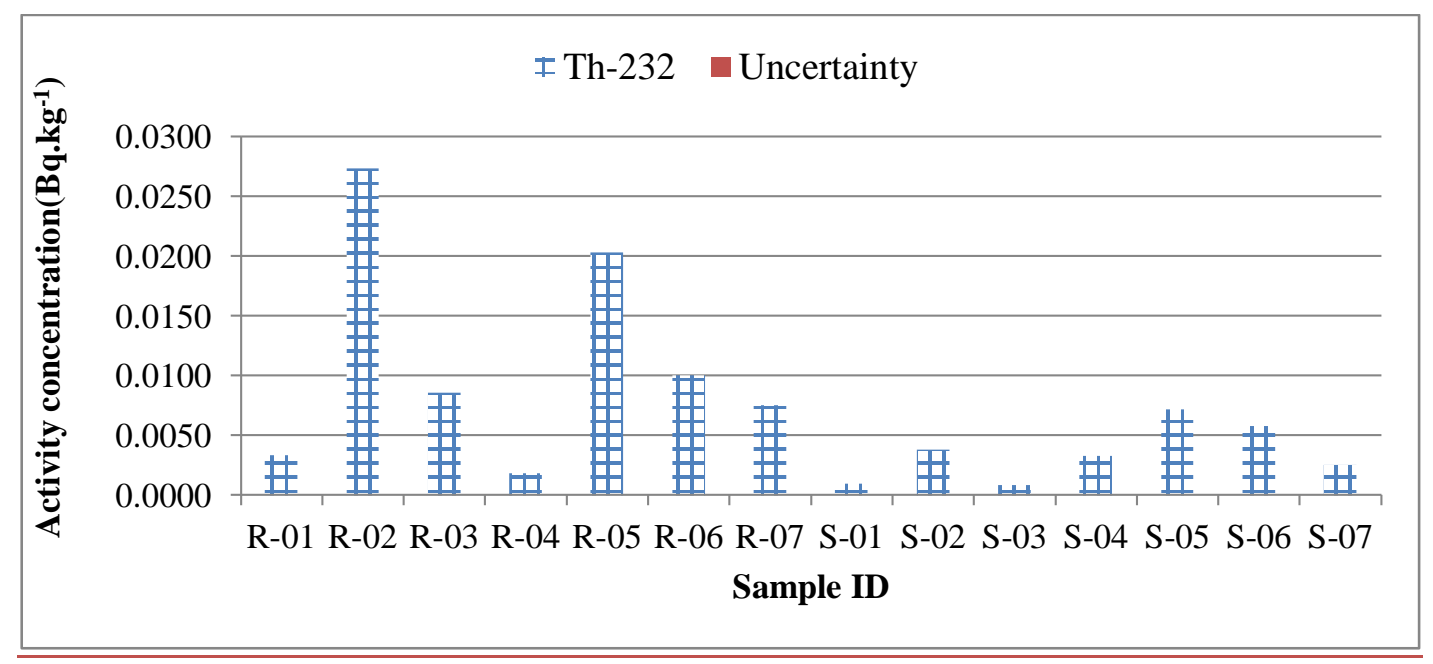

Fig. 4: Comparison of the activity concentrations of ${ }^{232} \mathrm{Th}$ of all the fish samples. 
88 Akhi Das Gupta, Md. Kowsar Alam, Shyamal Ranjan Chakraborty, A. K. M. Rezaur Rahman, S. I. Bhuian, Masud Kamal, Hasan Murad and Arun Kumar Deb

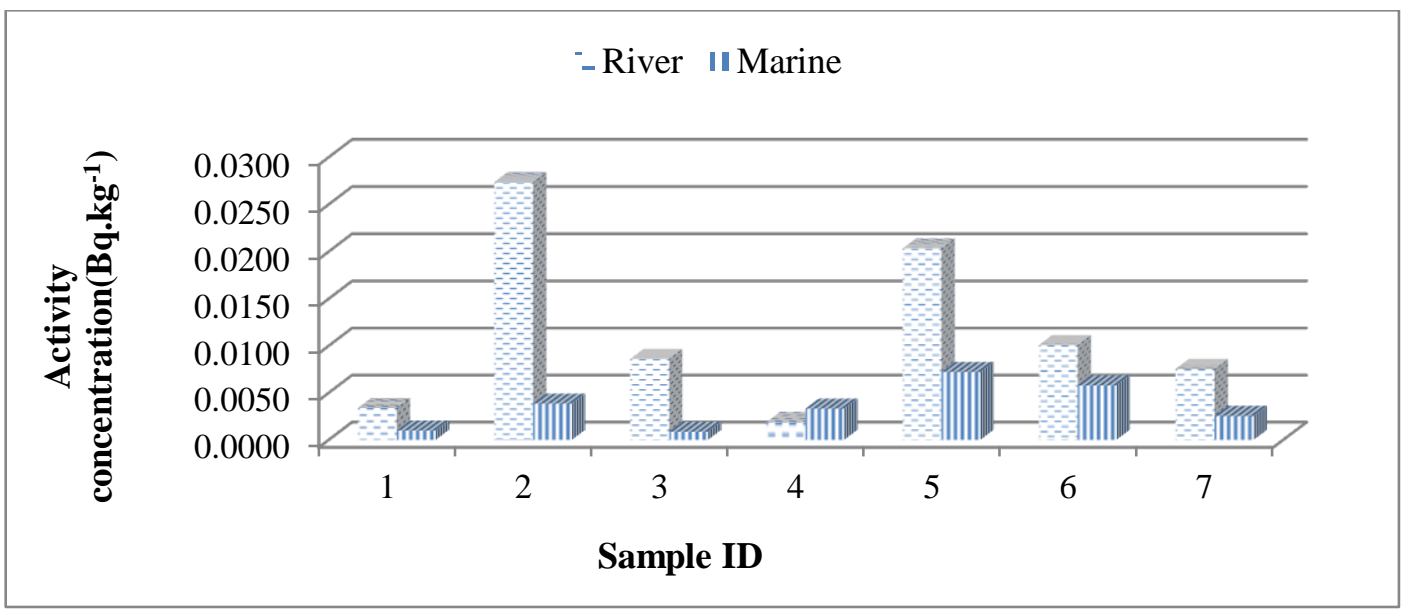

Fig. 5: Comparison of activity concentration of ${ }^{232} \mathrm{Th}$ between river (Karnaphuli estuary) and marine fish samples.

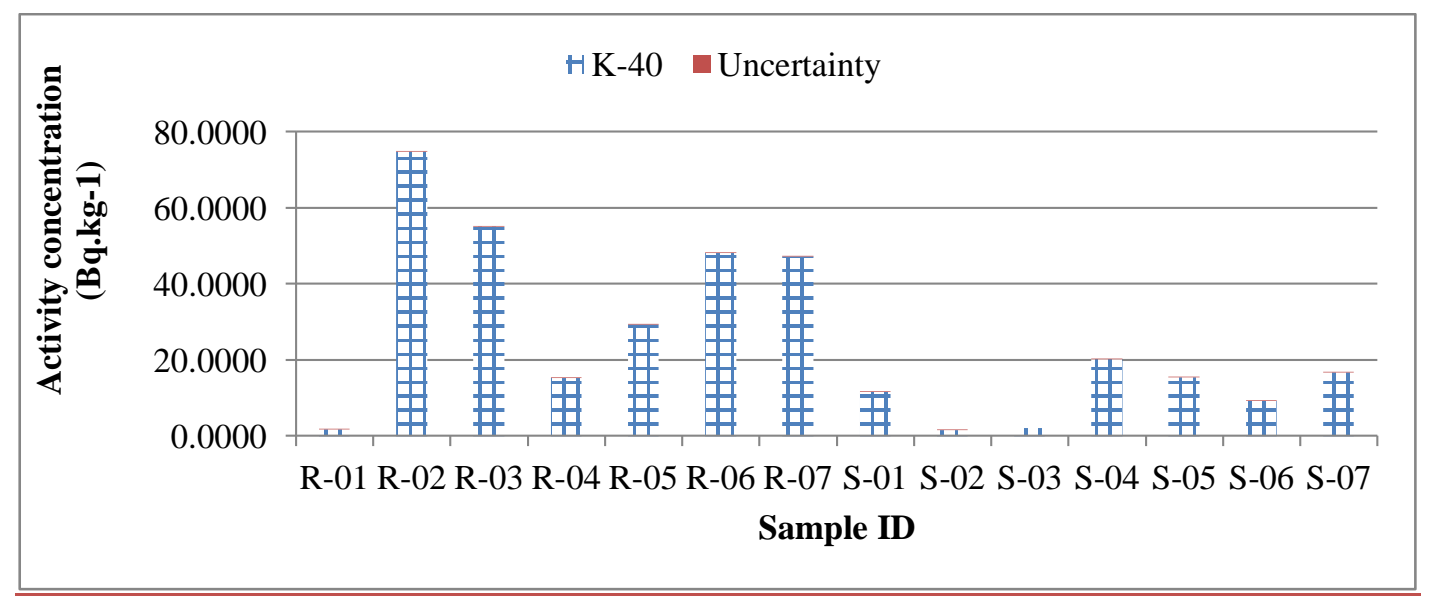

Figure. 6: Comparison of the activity concentrations of $40 \mathrm{~K}$ of all the fish samples. 
Effect of Fukushima Accident on Fishes and Fish Like other Foods Collected from the Bay of Bengal and Karnaphuli River, Bangladesh

Table 2: Comparison of present results with corresponding world average values and other study

\begin{tabular}{|c|c|c|c|c|c|}
\hline Country & Reference & $\begin{array}{c}{ }^{238} \mathrm{U} \\
\left(\mathrm{Bq} \cdot \mathrm{kg}^{-1}\right)\end{array}$ & $\begin{array}{c}{ }^{228} \mathrm{Ra} \\
\left(\mathrm{Bq} \cdot \mathrm{kg}^{-1}\right)\end{array}$ & $\begin{array}{c}{ }^{232} \mathrm{Th} \\
\left(\mathrm{Bq} \cdot \mathrm{kg}^{-1}\right)\end{array}$ & $\begin{array}{c}{ }^{40} \mathrm{~K} \\
\left(\mathrm{~Bq} \cdot \mathrm{kg}^{-1}\right)\end{array}$ \\
\hline \multirow{3}{*}{ Bangladesh } & $\begin{array}{l}\text { Present } \\
\text { study }\end{array}$ & 0.6109 & - & 0.0074 & 24.95 \\
\hline & [19] & 1.05 & 0.77 & - & 61.5 \\
\hline & [21] & 0.26 & - & 0.45 & 109.11 \\
\hline \multirow[t]{2}{*}{ Nigeria } & [25] & $37.22 \pm 4.31$ & - & $94.82 \pm 3.82$ & $\begin{array}{c}618.2 \pm \\
26.81\end{array}$ \\
\hline & [25] & $25.6 \pm 7.4$ & - & $52.4 \pm 28.7$ & $426 \pm 80$ \\
\hline Pakistan & [26] & $1.3 \pm 0.3$ & $1.0 \pm 0.2$ & - & $90 \pm 15$ \\
\hline \multirow{2}{*}{ Monaco } & [27] & 1.112 & - & 0.029 & 480 \\
\hline & [28] & $0.487 \pm 0.011$ & - & $0.0715 \pm 0.002$ & - \\
\hline \multirow{2}{*}{ Greenland } & [29] & - & - & - & 127 \\
\hline & [30] & 0.004 & - & 0.001 & - \\
\hline Brazil & [31] & 0.055 & - & 0.026 & - \\
\hline Worldwide & {$[16]$} & 0.008 & - & 0.0007 & 45 \\
\hline
\end{tabular}


90 Akhi Das Gupta, Md. Kowsar Alam, Shyamal Ranjan Chakraborty, A. K. M. Rezaur Rahman, S. I. Bhuian, Masud Kamal, Hasan Murad and Arun Kumar Deb

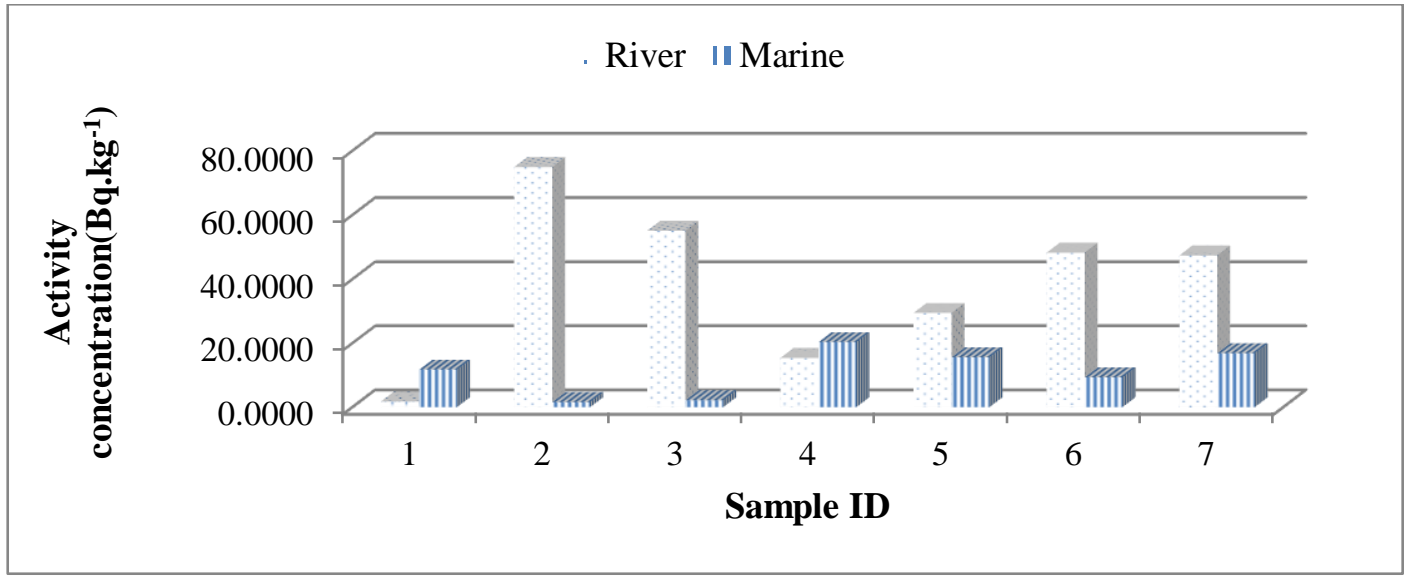

Fig. 7: Comparison of activity concentration of ${ }^{40} \mathrm{~K}$ between river (Karnaphuli estuary) and marine fish samples.

\subsection{Radiological Parameters or Radiation Hazard in the Fish samples:}

\subsubsection{Effective dose rate for fish samples:}

The mean value of the annual effective dose has been found to be $0.1 \mathrm{mSv} \cdot \mathrm{y}^{-1}$. The values of the effective dose for all samples obtained were lower than that of the world-wide average of $0.3 \mathrm{mSv} \cdot \mathrm{y}^{-1}$ [16]. The comparative data of the effective dose rate for all the samples are given in table 3.

\subsubsection{Internal hazard index, $\mathrm{H}_{\mathrm{int}}$ :}

The internal hazard index of these fish samples has been in the range from $0.0013 \pm$ 0 to $0.0291 \pm 0.0001$ with an average value of $0.0085 \pm 0$, which is less than the permissible limit $1(22,32)$. The results for all the samples are shown in the table 3 . 
Effect of Fukushima Accident on Fishes and Fish Like other Foods Collected from the Bay of Bengal and Karnaphuli River, Bangladesh

Table 3: The comparative data of the radiological parameters for the all fish samples

\begin{tabular}{|c|c|c|}
\hline Sample no & Internal hazard index, $\mathbf{H}_{\text {int }}$ & Effective dose $\left(\mathrm{Sv}_{\mathrm{y}} \mathrm{y}^{-1}\right)$ \\
\hline R-01 & $0.0016 \pm 0$ & $7.14 \times 10^{-6}$ \\
\hline $\mathrm{R}-02$ & $0.0291 \pm 0.0001$ & $3.03 \times 10^{-4}$ \\
\hline $\mathrm{R}-03$ & $0.0154 \pm 0$ & $2.23 \times 10^{-4}$ \\
\hline $\mathrm{R}-04$ & $0.0041 \pm 0$ & $6.20 \times 10^{-5}$ \\
\hline $\mathrm{R}-05$ & $0.0143 \pm 0$ & $1.19 \times 10^{-4}$ \\
\hline $\mathrm{R}-06$ & $0.0140 \pm 0$ & $1.95 \times 10^{-4}$ \\
\hline $\mathrm{R}-07$ & $0.0144 \pm 0$ & $1.91 \times 10^{-4}$ \\
\hline S-01 & $0.0030 \pm 0$ & $4.72 \times 10^{-5}$ \\
\hline S-02 & $0.0014 \pm 0$ & $6.28 \times 10^{-6}$ \\
\hline S-03 & $0.0013 \pm 0$ & $8.64 \times 10^{-6}$ \\
\hline S-04 & $0.0059 \pm 0$ & $8.21 \times 10^{-5}$ \\
\hline S-05 & $0.0060 \pm 0$ & $6.29 \times 10^{-5}$ \\
\hline S-06 & $0.0044 \pm 0$ & $3.76 \times 10^{-5}$ \\
\hline S-07 & $0.0045 \pm 0$ & $6.81 \times 10^{-5}$ \\
\hline Mean & $0.0085 \pm 0$ & $1.01 \times 10^{-4}$ \\
\hline
\end{tabular}

\section{Discussion}

The present study shows the concentrations of $U$ and Th -series radionuclides' $\left({ }^{232} \mathrm{Th},{ }^{238} \mathrm{U}\right)$ and ${ }^{40} \mathrm{~K}$ in the River (Karnaphuli estuary) and Marine fishes and fish like samples. The specific activity concentrations of $U$ and $T h$-series in all fish samples were found to be higher than that of the world average.

The mean activity concentration of ${ }^{238} \mathrm{U}$ in all samples were higher than those of Brazil [31], Ireland [30], England [28], other study [21] and lower than those of Nigeria [22], Monaco [27]. Again the activity concentrations of ${ }^{238} U$ in River 
92 Akhi Das Gupta, Md. Kowsar Alam, Shyamal Ranjan Chakraborty, A. K. M. Rezaur Rahman, S. I. Bhuian, Masud Kamal, Hasan Murad and Arun Kumar Deb

samples are found to be higher than the activity concentration of ${ }^{238} \mathrm{U}$ in marine samples.

The mean specific activity of ${ }^{232}$ Th in all samples were found to be higher than those of Ireland [30] and lower than those of England [28], Nigeria [22], Brazil [31], Monaco [27], other study [21]. Again the activity concentrations of ${ }^{232} \mathrm{Th}$ in River samples were found to be higher than that of the activity concentration of ${ }^{232} \mathrm{Th}$ in marine samples. The mean activity concentrations of ${ }^{40} \mathrm{~K}$ in the all samples are found to be lower than those of Nigeria [22], Monaco [27], and other study [21]. Again the specific activities of ${ }^{40} \mathrm{~K}$ in River samples are found to be higher than that of the specific activity of ${ }^{40} \mathrm{~K}$ in marine samples.

However, the activity concentration of ${ }^{232} \mathrm{Th},{ }^{238} \mathrm{U},{ }^{40} \mathrm{~K}$ in Crab was found to be higher than that of the activity concentrations of ${ }^{232} \mathrm{Th},{ }^{238} \mathrm{U},{ }^{40} \mathrm{~K}$ in Flesh. All the values of effective dose due to intake of radionuclides ingestion in humans obtained are lower than the world-wide average annual effective dose [17]. Moreover, the recommended value of the internal radiation hazard index $\mathrm{H}_{\text {int }}$ is one $[3,22]$ but in all samples the internal radiation hazard is less than one. The mean internal radiation hazard index is found to be 0.0085 . So the radioactive exposure from Marine and River (Karnaphuli estuary) fishes and other fish like food due to Fukushima nuclear accident does not pose any significant radiological threat to the population who are the consumer of the fishes of the present study areas. 


\section{Conclusions}

The following are the consequences of the present study.

$>$ The activity concentration of natural radionuclides of Uranium series has been found to be higher than that of the Thorium series in the entire fish samples.

$>$ The activity concentration of ${ }^{238} \mathrm{U}$ has been found to be high in river (Karnaphuli estuary) fish samples than Marine fish samples.

$>$ A large variation (1.5516 to $74.8658 \mathrm{~Bq} \cdot \mathrm{kg}^{-1}$ ) of the activity concentration of ${ }^{40} \mathrm{~K}$ is found in these samples.

$>$ The Radiological parameters viz. annual effective dose and internal hazard index in River (Karnaphuli estuary) fish sample is higher than the Marine fish samples.

$>$ The observed average internal radiation hazard index in the entire fish sample is 0.008529 , but the recommended value of the radiation hazard index is 1 (one).

Since the radiation hazard index is less than that of the standard value, so the population who are the consumer of this food are under the safety limit of radiological threat due to the dumping of the Fukushima nuclear accidental wastes in the Pacific ocean.

\section{References}

[1] K. R. Rao: Radioactive waste; Current Science 2001, 81(4), 1534.

[2] UNSCEAR: "United Nations Scientific Committee on The Effects of Atomic Radiation, UNSCEAR Report to the General Assembly with Scientific Annexes", United Nations, New York, 2000, 1, p. 1. 
94 Akhi Das Gupta, Md. Kowsar Alam, Shyamal Ranjan Chakraborty, A. K. M. Rezaur Rahman, S. I. Bhuian, Masud Kamal, Hasan Murad and Arun Kumar Deb

[3] International Atomic Energy Agency IAEA: "IAEA Technical Report series295", Section-2, 3, 1989.

[4] Sheila M. Innis: The Journal of Nutrition 2007, 137(4), 855. doi: 10.1093/jn/137.4.855

[5] Ricardo Uauy, Dennis R. Hoffman, Patricio Peirano, David G. Birch, Eileen E. Birch: Lipids, 2001, 36(9), 885. doi :10.1007/s11745-001-0798-1

[6] Danielle Swanson, Robert Block, and Shaker A. Mousa: Advances in Nutrition 2012, 3(1), 1. doi: 10.3945/an.111.000893

[7] Shane Bilsborough, and Neil Mann: International Journal of Sport Nutrition and Exercise Metabolism 2006, 16(2), 129. doi: 10.1123/ijsnem.16.2.129

[8] Guoyao Wu: Food \& Function 2016, 3(7), 1251. doi: 10.1039/c5fo01530h

[9] K. Brown, A. Halls, W. N. Adger, D. Conway, E. H. Allison, J. D. Reynolds, M. C. Badjeck, N. K. Dulvy, and A. Perry: "Marine Resources Assessment Group, London, UK, Fisheries Management Science Programme MRAG/DFID”, Project No. R4778J, 2005, p.169.

[10] M. M. Hussain: "Status of development of the Fishery and seafood processing Industry in Bangladesh", Special Bulletin, 1994.

[11] UNEP, FAO, IOC: Blue Carbon, The role of healthy oceans in binding carbon, 2009.

[12] Intergovernmental Oceanographic Commission of UNESCO: "International Geosphere-Biosphere Programme", Marine Environment Laboratories (MEL) of the International Atomic Energy Agency Scientific Committee on Oceanic Research, 2008.

[13] Manado Ocean Declaration: World Ocean Conference Ministerial/High Level Meeting, Manado, Indonesia, 11-14 May, 2009.

[14] PACFA : "Fisheries and Aquaculture in a Changing Climate", 2009. 
Effect of Fukushima Accident on Fishes and Fish Like other Foods Collected from the Bay of Bengal and Karnaphuli River, Bangladesh

[15] FAO: "Report of the FAO Expert Workshop on Climate Change Implications for Fisheries and Aquaculture", FAO Fisheries Report No. 870, Rome, Italy, 2008.

[16] TEPCO press release: TEPCO, Retrieved 24 May, 2012.

[17] "TEPCO puts radiation release early in Fukushima crisis at $900 \mathrm{PBq}$, Kyodo News, Archived from the original on 8 July 2012”, Retrieved 24 May, 2012.

[18] Kevin Krolicki: "Fukushima radiation higher than first estimated", Reuters, Retrieved 24 May, 2012.

[19] A. El-Taher: Radiation Protection Dosimetry, 2010, 138(2), 166.

[20] M. K. Alam, S. R. Chakraborty, A. K. M. Rezaur Rahman, A. K. Deb, M. Kamal, M. I. Chowdhury, M. S. Uddin: Radiation Protection Dosimetry, 2013, 153(3), 316. doi:10.1093/rpd/ncs117

[21] UNSCEAR, "United Nations Scientific Committee on the Effects of Atomic Radiation, Exposure from Natural Sources of Radiation: UNSCEAR Report to the General Assembly, with annexes"; United Nations, New York, 1988.

[22] ICRP, Radionuclide Transformations, Publication of International Commission on Radiological Protection Publication, 1983, 11-13, 38.

[23] T. Jabbar, K. Khan, M. S. Subhani, P. Akhter, A. Jabbar: Radiation Protection Dosimetry, 2008, 132(1), 88.

[24] S. Ghose, M. N. Alam, M. N. Islam: Radiation Protection Dosimetry, 2000, 87(4), 287.

[25] O. Sowole and K. W. Giwa: International Journal of Science and Technology $20132(6), 438$.

[26] A. S. Mollah: Institute of Nuclear Science and Technology, Ganakbari, Savar, Bangladesh, 1996. Available at http://www.irpa.net/irpa9/cdrom/VOL.2/V2_154.PDF 
96 Akhi Das Gupta, Md. Kowsar Alam, Shyamal Ranjan Chakraborty, A. K. M. Rezaur Rahman, S. I. Bhuian, Masud Kamal, Hasan Murad and Arun Kumar Deb

[27] R. Adamu, Y. I. Zakari, A. Y. Ahmed, S. Abubakar. and A. M. Vatsa: Pelagia Research Library Advances in Applied Science Research, 2013, 4(4), 283.

[28] R. Macholz: Molecular Nutrition \& Food Research, 1988, 33(2), 152. doi: 10.1002/food.19890330208

[29] J. Beretka and P. J. Mathew: Health Physics, 1985, 48(1), 87.

[30] J. A. Ademola, and S. I. Ehiedu: African Journal of Biomedical Research, 2010, 13(2), 99.

[31] S. N. A. Tahir, A. S. Alaamer, M. Ayub, M. Z. Khan: Health Physics, 2010, 98(5), 741. doi: 10.1097/HP.0b013e3181d18f4a

[32] M. K. Pham, J. La Rosa, S. -H. Lee, P. P. Povinec: “IAEA/AL/145, IAEA/MEL/73, IAEA”, Marine Environment Laboratory, Monaco, 2004.

[33] A. K. Young, D. McCubbin, W. C. Camplin: "Lowestoft Laboratory, Environment Report RL17/02", Lowestoft, Suffolk, NR33 0HT, England, UK, 2002.

[34] G. Kanisch, and M. -O. Aust: Biogeosciences, 2013, 10, 5399. doi:10.5194/bg$10-5399-2013$

[35] Barry R. Friedlander, Michael Gochfeld, Joanna Burger, and Charles W. Powers: A CRESP Science Review, 2005.

[36] Wagner de S. Pereira, Alphonse Kelecom, and Delcy de A. Py Júnior: Brazilian Journal of Oceanography, 2010, 58(spe4) (special issue IICBBM), 25. doi: 10.1590/S1679-87592010000800005

[37] 1990 Recommendations of the International Commission on Radiological Protection: ICRP Publication 60, 1991, 21 (1-3), 1.

Manuscript received on 07 August, 2017, Revised manuscript received on 10 June, 2018 and accepted on 27 June, 2018

The Chittagong Univ. J. Sc. Vol. 40, 2018 